\title{
An Exactly Solvable Model of Interacting Bosons
}

\author{
A. B. Balantekin* \\ Department of Physics, University of Wisconsin; \\ Madison, Wisconsin 53706 USA \\ T. Dereli ${ }^{\dagger}$ \\ Department of Physics, Koç University; \\ 34450 Sarlyer-İstanbul, Turkey \\ Y. Pehlivan ${ }^{\ddagger}$ \\ Department of Physics, Middle East Technical University; \\ 06531 Ankara, Turkey
}

\begin{abstract}
We introduce a class of exactly solvable boson models. We give explicit analytic expressions for energy eigenvalues and eigenvectors for an sd-boson Hamiltonian, which is related to the $S O(6)$ chain of the Interacting Boson Model Hamiltonian.
\end{abstract}

\section{INTRODUCTION}

A number of complex quantum systems can be described algebraically using bosonic degrees of freedom. For example even-even nuclei can be described in terms of a system of one boson with angular momentum $L=0$, called $s$, and five components of another boson with angular momentum $L=2$, called $d_{\mu}, \mu=0, \pm 1, \pm 2$. This model, called Interacting Boson Model, has been very successful in describing the low-lying collective states of eveneven nuclei $[1,2]$ A similar boson model, using an angular momentum $L=1(p)$ boson in addition to the $s$-boson has been used to describe the structure of molecules [3] and hadrons [4]. The s-d boson model has an $S U(6)$ symmetry and the s-p boson model has an $S U(4)$

*Electronic address: baha@nucth.physics.wisc.edu

$\dagger$ Electronic address: tdereli@ku.edu.tr

${ }^{\ddagger}$ Electronic address: yamac@gursey . gov . tr 
symmetry. Analytic solutions of these models are given whenever the Hamiltonians can be written in terms of the Casimir operators of the $S U(6)$ (or $S U(4)$ ) group and its subgroups. In particular for the $S O(6)$ chain the Hamiltonian contains the boson pairing operator.

Since the boson creation operators $d_{\mu}^{\dagger}$ are spherical tensors and the annihilation operators can be written as spherical tensors after the introduction of a suitable phase (i.e. $\tilde{d}_{\mu}=$ $(-1)^{\mu} d_{-\mu}$ ) we will use the dot product notation of spherical tensors, $(\cdot)$, below. It is easy to show that the three operators

$$
\begin{aligned}
S^{+} & =\frac{1}{2}\left(d^{\dagger} \cdot d^{\dagger}\right), \\
S^{-} & =\frac{1}{2}(\tilde{d} \cdot \tilde{d}),
\end{aligned}
$$

and

$$
S^{0}=\frac{1}{4} \sum_{\mu}\left(d_{\mu}^{\dagger} d_{\mu}+d_{\mu} d_{\mu}^{\dagger}\right),
$$

generate the d-boson quasi-spin group $S U(1,1)$. This group can also be represented using only the s- or p-bosons. The eigenvalues of the operator $S^{0}$ are given by $(n / 2)+(1+\alpha) / 4$, where $n$ is the total number of the bosons and the quantity $\alpha$ is 0,1 , and 2 for the s-,p-, and d-bosons, respectively. For the $S O(6)$ symmetry chain one needs to include both the sand d- bosons in the realization of the algebra:

$$
\begin{gathered}
S^{+}=\frac{1}{2}\left[\left(d^{\dagger} \cdot d^{\dagger}\right)-\left(s^{\dagger} s^{\dagger}\right)\right], \\
S^{-}=\frac{1}{2}[(\tilde{d} \cdot \tilde{d})-(s s)],
\end{gathered}
$$

and

$$
S^{0}=\frac{1}{4}\left[\sum_{\mu}\left(d_{\mu}^{\dagger} d_{\mu}+d_{\mu} d_{\mu}^{\dagger}\right)+\left(s^{\dagger} s+s s^{\dagger}\right)\right] .
$$

The pairing operator of the $S O(6)$ limit is then $P_{6}=S^{+} S^{-}$, where $S^{ \pm}$are given by Eqs. (1.4) and (1.5).

Our goal in this paper is to consider a system of s- and d-bosons near, but not necessarily at the $S O(6)$ limit. It is worth to point out that similar efforts were undertaken by others. In Ref. [5] the $U(5)$ to $S U(3)$ transition in the interacting boson model was considered. In Ref. [6] an exactly solvable model of bosons with a repulsive pairing and single-particle energies proportional to the angular momentum quantum number was introduced. This model was further elaborated in Ref. [7]. In these models the eigenvalues and the eigenfunctions are written down in terms of variables that need to be numerically evaluated by solving nonlinear equations. Our aim here is to introduce a model for which an explicit analytic energy level expression can be given for all levels and analytic expressions can be given for at least few lowest eigenstates. A preliminary version of this work was presented in Ref. [8]. 


\section{THE MODEL}

To present our model we first introduce the operator

$$
S^{+}(\lambda)=\frac{1}{2}\left[\frac{\left(d^{\dagger} \cdot d^{\dagger}\right)}{1-\lambda}-\frac{s^{\dagger} s^{\dagger}}{\lambda}\right] .
$$

Clearly $S^{+}(\lambda)$ is proportional to the operator in Eq. (1.4) when the parameter $\lambda=1 / 2$. However it represents a general mixing of $s$ and $d$ bosons when $\lambda \neq 1 / 2$. Similarly we introduce the operators

$$
S^{-}(\lambda)=\frac{1}{2}\left[\frac{(\tilde{d} \cdot \tilde{d})}{1-\lambda}-\frac{s s}{\lambda}\right]
$$

and

$$
S^{0}(\lambda)=\frac{1}{4}\left[\frac{\sum_{\mu}\left(d_{\mu}^{\dagger} d_{\mu}+d_{\mu} d_{\mu}^{\dagger}\right)}{1-\lambda}-\frac{\left(s^{\dagger} s+s s^{\dagger}\right)}{\lambda}\right] .
$$

In this paper we show that the eigenvalues and the eigenstates of the Hamiltonian

$$
\mathcal{H}=-\frac{1}{4}\left[\frac{\hat{N}_{d}}{1-\lambda}-\frac{\hat{N}_{s}}{\lambda}\right]^{2}+\frac{1}{2}\left[\frac{\hat{N}_{d}}{(1-\lambda)^{2}}+\frac{\hat{N}_{s}}{\lambda^{2}}\right]-W(\lambda)\left[\frac{\hat{N}_{d}}{1-\lambda}-\frac{\hat{N}_{s}}{\lambda}\right]+S^{+}(\lambda) S^{-}(\lambda),
$$

can be analytically calculated. Here $\hat{N}_{d}$ and $\hat{N}_{s}$ are the d- and s-boson number operators, and the function $W(\lambda)$ is defined below.

The operators given in Eqs. (1.1), (1.2), and (1.3) and their s-boson counterparts satisfy the $S U(1,1)$ commutation relations

$$
\left[S_{i}^{+}, S_{j}^{-}\right]=-2 \delta_{i j} S_{j}^{0},
$$

and

$$
\left[S_{i}^{0}, S_{j}^{ \pm}\right]= \pm \delta_{i j} S_{j}^{ \pm},
$$

where $i, j$, etc. represent different $S U(1,1)$ algebras written using only the s,d, etc. bosons. Using Eqs. (2.5) and (2.6) we obtain the commutation relations

$$
\begin{aligned}
& {\left[S^{+}(\lambda), S^{-}(\mu)\right]=-2 \frac{S^{0}(\lambda)-S^{0}(\mu)}{\lambda-\mu},} \\
& {\left[S^{0}(\lambda), S^{ \pm}(\mu)\right]= \pm \frac{S^{ \pm}(\lambda)-S^{ \pm}(\mu)}{\lambda-\mu},} \\
& {\left[S^{0}(\lambda), S^{0}(\mu)\right]=\left[S^{ \pm}(\lambda), S^{ \pm}(\mu)\right]=0 .}
\end{aligned}
$$

Eqs. (2.8) were first studied by Gaudin [9] in the context of interacting spins on a lattice. The limit of these commutators when $\lambda=\mu$ can easily shown to be 


$$
\begin{aligned}
& {\left[S^{+}(\lambda), S^{-}(\lambda)\right]=-2 \frac{\delta S^{0}(\lambda)}{\delta \lambda},} \\
& {\left[S^{0}(\lambda), S^{ \pm}(\lambda)\right]= \pm \frac{\delta S^{ \pm}(\lambda)}{\delta \lambda}}
\end{aligned}
$$

Next it is easy to show that the parametric Hamiltonians

$$
H(\lambda)=S^{0}(\lambda) S^{0}(\lambda)-\frac{1}{2} S^{+}(\lambda) S^{-}(\lambda)-\frac{1}{2} S^{-}(\lambda) S^{+}(\lambda)
$$

form a one-parameter family of mutually commuting operators:

$$
[H(\lambda), H(\mu)]=0 .
$$

Starting from a lowest weight vector and using $J^{+}(\lambda)$ as step operators, one can diagonalize them simultaneously. Lowest weight vector $\mid 0>$ is the boson vacuum:

$$
S^{-}(\lambda) \mid 0>=0 .
$$

Note that our choice of the ground state is different from what is normally taken in the $\mathrm{SO}(6)$ limit, which has the maximum population of the s-bosons. The function $W(\lambda)$ we introduced earlier in the Hamiltonian of Eq. (2.4) is obtained by the action of the operator $S^{0}(\lambda)$ on the vacuum state:

$$
S^{0}(\lambda)|0>=W(\lambda)| 0>.
$$

For the s-d boson model $W(\lambda)$ takes the form

$$
W(\lambda)=\frac{1}{4}\left[\frac{5}{1-\lambda}-\frac{1}{\lambda}\right] .
$$

The boson vacuum itself is an eigenvector of $H(\lambda)$ :

$$
H(\lambda)\left|0>=\epsilon_{0}(\lambda)\right| 0>
$$

with the eigenvalue

$$
\epsilon_{0}(\lambda)=W(\lambda)^{2}-W^{\prime}(\lambda) .
$$

It can be shown that a vector of the form

$$
\left|\xi>\equiv S^{+}(\xi)\right| 0>
$$

is also an eigenvector of $H(\lambda)$ if $\xi$ the solution of

$$
W(\xi)=0 .
$$

In this case the eigenvalue is given by 


$$
\epsilon_{1}(\lambda)=\epsilon_{0}(\lambda)-2 \frac{W(\lambda)}{\lambda-\xi}
$$

Using the algebra given in Eqs. (2.8) it is easy to show that the state

$$
\left|\xi_{1}, \xi_{2}, \ldots, \xi_{n}>\equiv S^{+}\left(\xi_{1}\right) S^{+}\left(\xi_{2}\right) \ldots S^{+}\left(\xi_{n}\right)\right| 0>
$$

is an eigenvector of $H(\lambda)$ if the quantities $\xi_{1}, \xi_{2}, \ldots$ satisfy the following system of equations:

$$
W\left(\xi_{\alpha}\right)=\sum_{\beta \neq \alpha}^{n} \frac{1}{\xi_{\alpha}-\xi_{\beta}} \quad \text { for } \quad \alpha=1,2, \ldots, n .
$$

The eigenvalue of the Hamiltonian in Eq. (2.12) on the state of Eq. (2.22) is given by

$$
\epsilon_{n}(\lambda)=\epsilon_{0}(\lambda)-2 \sum_{\alpha=1}^{n} \frac{W(\lambda)-W\left(\xi_{\alpha}\right)}{\lambda-\xi_{\alpha}} .
$$

Comparing the Hamiltonians of Eq. (2.4) and (2.12) and using Eqs. (2.11), it follows that the energy eigenvalues of the Hamiltonian $\mathcal{H}$ are given by

$$
E_{n}(\lambda)=2 \sum_{\alpha=1}^{n} \frac{W(\lambda)-W\left(\xi_{\alpha}\right)}{\lambda-\xi_{\alpha}}
$$

All the above considerations are equally applicable to both real and complex values of $\lambda$. However only when $\lambda$ is real then our Hamiltonian is Hermitian with real eigenvalues. This is true even when the solutions of Eq. (2.23) are complex.

\section{CALCULATION OF THE EIGENVALUES AND THE EIGENFUNCTIONS}

In order to evaluate energy eigenvalues and eigenfunctions we will use the general expression

$$
W(\lambda)=\left[\frac{A}{a_{1}-\lambda}+\frac{B}{a_{2}-\lambda}\right]
$$

so that our calculations are equally applicable to the mixture of other bosons. Inserting Eq. (3.1) into Eq. (2.25) it is easy to show that

$$
E_{n}(\lambda)=2 \sum_{\alpha=1}^{n}\left[\frac{A}{\left(a_{1}-\lambda\right)\left(a_{1}-\xi_{\alpha}\right)}+\frac{B}{\left(a_{2}-\lambda\right)\left(a_{2}-\xi_{\alpha}\right)}\right] .
$$

It turns out that an expression for energy eigenvalues can be obtained without explicitly calculating the solutions of Eq. (2.23). From Eq. (2.23) it is fairly straightforward to show that

$$
\sum_{\alpha=1}^{n} W\left(\xi_{\alpha}\right)=0
$$

Similarly considering the expression 


$$
\sum_{\alpha=1}^{n} \xi_{\alpha} W\left(\xi_{\alpha}\right)=\sum_{\alpha=1}^{n}\left[\sum_{\beta<\alpha} \frac{\xi_{\alpha}}{\xi_{\alpha}-\xi_{\beta}}+\sum_{\beta>\alpha} \frac{\xi_{\alpha}}{\xi_{\alpha}-\xi_{\beta}}\right]
$$

and interchanging the dummy indices $\alpha$ and $\beta$ in the last sum one finds that

$$
\sum_{\alpha=1}^{n} \xi_{\alpha} W\left(\xi_{\alpha}\right)=\sum_{(\alpha, \beta) \text { pairs }}^{n} \frac{\xi_{\alpha}-\xi_{\beta}}{\xi_{\alpha}-\xi_{\beta}}=\frac{n(n-1)}{2} .
$$

Using Eqs. (3.1) and (3.3) one then gets

$$
A \sum_{\alpha=1}^{n} \frac{1}{a_{1}-\xi_{\alpha}}=-B \sum_{\alpha=1}^{n} \frac{1}{a_{2}-\xi_{\alpha}} .
$$

Inserting Eq. (3.1) into Eq. (3.5) and employing the identity

$$
\frac{\xi_{\alpha}}{a-\xi_{\alpha}}=\frac{a}{a-\xi_{\alpha}}-1
$$

we get

$$
A \sum_{\alpha=1}^{n}\left(\frac{a_{1}}{a_{1}-\xi_{\alpha}}-1\right)+B \sum_{\alpha=1}^{n}\left(\frac{a_{2}}{a_{2}-\xi_{\alpha}}-1\right)=\frac{n(n-1)}{2} .
$$

Using Eq. (3.6) in Eq. (3.8) one then obtains

$$
B\left(a_{2}-a_{1}\right) \sum_{\alpha=1}^{n} \frac{1}{a_{2}-\xi_{\alpha}}=\frac{n(n-1)}{2}+(A+B) n .
$$

Using Eq. (3.6), the energy eigenvalues of Eq. (3.2) take the form

$$
E_{n}(\lambda)=2 B\left(\frac{1}{a_{2}-\lambda}-\frac{1}{a_{1}-\lambda}\right) \sum_{\alpha=1}^{n} \frac{1}{a_{2}-\xi_{\alpha}} .
$$

Finally inserting Eq. (3.9) into Eq. (3.10) we obtain

$$
E_{n}(\lambda)=\frac{-2}{\left(a_{1}-\lambda\right)\left(a_{2}-\lambda\right)}\left[\frac{n(n-1)}{2}+(A+B) n\right] .
$$

In our model we took $a_{1}=1, a_{2}=0, A=5 / 4$, and $B=1 / 4$ resulting in

$$
E_{n}(\lambda)=\frac{1}{\lambda(1-\lambda)}\left[n^{2}+2 n\right]
$$

Even though we did not need explicit solutions of Eq. (2.23) to evaluate the energy eigenstates, we nevertheless need to find these solutions to be able to write down the energy eigenstates. To achive this goal we first introduce a change of variables

$$
\xi_{\alpha}=a_{2}+\zeta_{\alpha}\left(a_{1}-a_{2}\right) .
$$

Using these new variables the Bethe ansatz equation Eq. (2.23) takes the form 


$$
\sum_{\beta \neq \alpha}^{n} \frac{1}{\zeta_{\alpha}-\zeta_{\beta}}+\frac{B}{\zeta_{\alpha}}-\frac{A}{1-\zeta_{\alpha}}=0 \quad \text { for } \quad \alpha=1,2, \ldots, n,
$$

where $B=1 / 4$ and $A=5 / 4$ in the s-d boson problem. In Ref. [10], Stieltjes had shown that the polynomial

$$
p_{n}(\xi)=\prod_{\alpha=1}^{n}\left(\zeta-\zeta_{\alpha}\right)
$$

satisfies the hypergeometric differential equation

$$
x(1-x) p_{n}^{\prime \prime}(x)+[2 B-2(A+B)] p_{n}^{\prime}(x)+n(n+2 A+2 B-1) p_{n}(x)=0 .
$$

Hence $\zeta_{\alpha}$ are the roots of the polynomials

$$
\sum_{k} \frac{(-n)_{k}(n+2 A+2 B-1)_{k}}{(2 B)_{k} k !} x^{k},
$$

where

$$
(a)_{n}=a(a+1) \cdots(a+n-1)
$$

for $n=1,2, \cdots$ and $(a)_{0}=0$. So for example the first excited eigenstate is

$$
\frac{A+B}{2}\left[\frac{\left(d^{\dagger} \cdot d^{\dagger}\right)}{A}-\frac{s^{\dagger} s^{\dagger}}{B}\right] \mid 0>,
$$

and the second eigenstate is

$$
(A+B+1)^{2}\left[\frac{\left(d^{\dagger} \cdot d^{\dagger}\right)}{2 A+1-\gamma}-\frac{s^{\dagger} s^{\dagger}}{2 B+1+\gamma}\right]\left[\frac{\left(d^{\dagger} \cdot d^{\dagger}\right)}{2 A+1+\gamma}-\frac{s^{\dagger} s^{\dagger}}{2 B+1-\gamma}\right] \mid 0>,
$$

where

$$
\gamma=\sqrt{\frac{(2 A+1)(2 B+1)}{2 A+2 B+1}}
$$

Finding the eigenstates of the third, fourth, etc. excited states simply requires finding the roots of a cubic, quartic, etc. polynomial. Even though for the third and fourth order polynomials this can be done analytically, the resulting expressions are not particularly illuminating. Of course the roots can be calculated numerically for any order.

This work was supported in part by the U.S. National Science Foundation Grants No. INT-0352192 and PHY-0244384 at the University of Wisconsin, and in part by the University of Wisconsin Research Committee with funds granted by the Wisconsin Alumni Research Foundation. The research of YP was supported in part by the Turkish Scientific and Technical Research Council (TÜBITTAK). 


\section{REFERENCES}

[1] A. Arima and F. Iachello, Annals Phys. 99 (1976) 253.

[2] A. Arima and F. Iachello, Phys. Rev. Lett. 40 (1978) 385.

[3] F. Iachello and R.D. Levine, Int. J. Quantum Chem. 23 (1983) 1679.

[4] F. Iachello, Nucl. Phys. A 518 (1990) 173.

[5] F. Pan, J. P. Draayer, and Y. Luo, Phys. Lett. B 576 (2003) 297.

[6] J. Dukelsky and S. Pittel, Phys. Rev. Lett. 86, 4791 (2001) [arXiv:nucl-th/0102034].

[7] F. Pan, L. R. Dai, Y. A. Luo and J. P. Draayer, Phys. Rev. C 68 (2003) 014308.

[8] A. B. Balantekin, arXiv:nucl-th/0312072.

[9] M. Gaudin, J. Physique 37 (1976), 1087, M. Gaudin, La Fonction d'onde de Bethe, Collection du Commissariat a l'énergie atomique, Masson, Paris, 1983.

[10] T.J. Stieltjes, 1914 Sur Quelques Theoremess d'Algebre, Oeuvres Completes, V. 11 (Groningen:Noordhoff). 